\title{
Femtosecond-pulsed optical heating of gold nanoparticles
}

\author{
Guillaume Baffou* and Hervé Rigneault \\ Institut Fresnel, CNRS, Aix-Marseille Université, École Centrale de Marseille, \\ Domaine Universitaire Saint-Jérôme, 13397 Marseille, France
}

(Dated: March 10, 2011)

\begin{abstract}
We investigate theoretically and numerically the thermodynamics of gold nanoparticles immersed in water and illuminated by a femtosecond-pulsed laser at their plasmonic resonance. The spatiotemporal evolution of the temperature profile inside and outside is computed using a numerical framework based on a Runge-Kutta algorithm of the fourth order. The aim is to provide a comprehensive description of the physics of heat release of plasmonic nanoparticles under pulsed illumination, along with a simple and powerful numerical algorithm. In particular, we investigate the amplitude of the initial instantaneous temperature increase, the physical differences between pulsed and $\mathrm{cw}$ illuminations, the time scales governing the heat release into the surroundings, the spatial extension of the temperature distribution in the surrounding medium, the influence of a finite thermal conductivity of the gold/water interface, the influence of the pulse repetition rate of the laser, the validity of the uniform temperature approximation in the metal nanoparticle, and the optimum nanoparticle size (around 40nm) to achieve maximum temperature increase.
\end{abstract}

\section{INTRODUCTION}

Gold nanoparticles (NPs) can act as efficient nanosources of heat under visible or infrared illumination at the plasmonic resonance due to enhanced light absorption. ${ }^{1}$ The ability to locally heat at the nanoscale opens the path for new promising achievements in Nanotechnology and especially for nanoscale control of temperature distribution, ${ }^{2}$ chemical reactions, ${ }^{3}$ phase transition, ${ }^{4}$ material growth, ${ }^{5}$ photothermal cancer therapy $^{6-8}$ and drug release. ${ }^{9,10}$

The use of femtosecond-pulsed illumination on gold NPs expands the range of applications compared to continuous (cw) illumination. First, it can lead to nonlinear optical processes like two-photon luminescence or second harmonic generation with applications mainly in bio-imaging. ${ }^{11,12}$ Then, it can trigger a sudden temperature increase at the sub-nanosecond scale and subsequent effects such as acoustic waves used for opto-acoustic imaging $^{13,14}$ or bubble formation for nanosurgery. ${ }^{15} \mathrm{~A}$ sharp and brief temperature increase of a NP generated by a femtosecond laser can also contribute to confine the temperature increase at the close vicinity of the NP to avoid extended heating of the whole medium when not desired. ${ }^{16}$

Several experimental and numerical approaches aimed at studying the internal processes of heat generation under pulsed illumination and the subsequent effects observed in the surrounding medium, e.g. temperature and pressure variations, ${ }^{17-19}$ acoustic wave generation, ${ }^{18}$ vibration modes,${ }^{20-22}$, cell apoptosis, ${ }^{11}$ drug release ${ }^{9,23}$ nanosurgery, ${ }^{15,24}$ bubble formation, ${ }^{25-28}$ NP shape modification ${ }^{29}$ and melting, ${ }^{29-31}$ nanosecondpulses for biomedical applications, ${ }^{32,33}$ extreme thermodynamics conditions. ${ }^{33-37}$

In this paper, we present and use a versatile numerical framework to investigate theoretically and numerically the evolution of the temperature distribution of a gold nanoparticle immersed in water when shined by a femtosecond-pulsed laser. Various degrees of complexity exist to describe theoretically such a problem. We chose a progressive approach consisting in going from simple to more sophisticated: We shall start with the more ba- sic description of the problem, a point-like source of heat to model the NP, and then refine the description of the system by taking into account successive refinements, namely a finite-size structure, an gold/water interface conductivity and a non-uniform NP inner temperature. In each case, the physics and the associated constitutive equations are detailed and the approximations discussed. The aim is to answer all the questions related to characteristic time, space and temperature increase in fs-pulsed optical heating of gold NP.

Details regarding the numerical algorithm we developed are given in appendix.

\section{RESULTS AND DISCUSSION}

\section{A. Physical system}

We consider a system with spherical symmetry consisting of a gold nano-sphere of radius $R$ immersed in water (Fig. 1). This nanoparticle is uniformly illuminated by a laser light at its plasmonic resonance angular frequency $\omega=2 \pi c / \lambda_{0}=k c / n_{\mathrm{w}}, c$ is the speed of light

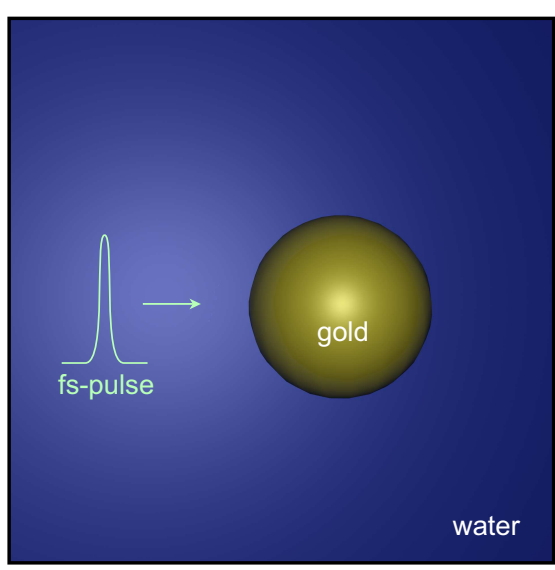

FIG. 1. Nature of the system investigated: A spherical gold nanoparticle immersed in water shined by a femtosecondpulsed laser. 
and $n_{\mathrm{w}}$ the optical index of water. No mass transfer like fluid convection or bubble formation is considered. We shall focus on moderate temperature increase (typically a few tens of degrees) and not consider extreme thermodynamic conditions. For this reason, all the parameters describing the materials (water and gold) are assumed to remain constant within the temperature range investigated. Whenever a femtosecond pulse is mentioned, it has to be understood as a pulse, the duration of which is smaller than the characteristic time of electron-phonon scattering $\tau_{\mathrm{e}-\mathrm{ph}} \sim 1.7 \mathrm{ps}$. For instance, this can correspond to the use of a Ti:Sapphire laser, which usually provides a pulse duration around $100 \mathrm{fs}$.

In the following, any mentioned temperature $T$ will have to be understood as a temperature increase above this initial ambient temperature.

Anywhere in the system, we can define the thermal energy density and thermal current density that read respectively $u_{\mathrm{th}}(r, t)=\rho c T(r, t)$ and $\mathbf{j}_{\mathrm{th}}(r, t)=$ $-\kappa \nabla T(r, t)$ where $\rho$ is the mass density, $c$ the specific heat capacity at constant pressure and $\kappa$ the thermal conductivity of the system at the position $r$. From the energy conservation equation $\partial_{t} u_{\mathrm{th}}(r, t)+\nabla \cdot \mathbf{j}_{\mathrm{th}}(r, t)=$ $p(r, t)$, on obtain the heat diffusion equation:

$$
\rho c \partial_{t} T(r, t)=\kappa \nabla^{2} T(r, t)+p(r, t)
$$

where $p(r, t)$ is the heat power density (nonzero only inside the NP $(r<R)$, where the light is absorbed),

For the system under consideration in this work, this yields a set of two differential equations, one for each medium (gold and water), along with two boundary conditions at the gold/water interface:

$$
\left\{\begin{array}{l}
\text { Diffusion equations: } \\
\rho_{\mathrm{Au}} c_{\mathrm{Au}} \partial_{t} T(r, t)=\kappa_{\mathrm{Au}} \nabla^{2} T(r, t)+p(r, t) \text { for } r<R \\
\rho_{\mathrm{w}} c_{\mathrm{w}} \partial_{t} T(r, t)=\kappa_{\mathrm{w}} \nabla^{2} T(r, t) \text { for } r>R \\
\text { Boundary conditions at } r=R: \\
\kappa_{\mathrm{w}} \partial_{r} T\left(R^{+}, t\right)=\kappa_{\mathrm{Au}} \partial_{r} T\left(R^{-}, t\right) \\
T\left(R^{+}, t\right)=T\left(R^{-}, t\right)
\end{array}\right.
$$

Au and w subscripts refer to gold and water. The first boundary condition ensures heat flux conservation at the NP interface.

It has been demonstrated experimentally that an interface resistivity at the gold/water interface exists and can play a significant role in the heat release. ${ }^{38-41}$ The interface resistivity can reach appreciable values when the liquid does not wet the solid. The wetting depends on the nature of the interface, and in particular a possible molecular coating. Namely, hydrophobic coatings are associated to poor thermal conductivities. The direct consequence of a finite interface conductivity $g$ (or resistivity $1 / g$ ) is a temperature drop/jump/discontinuity $\Delta T$ at the NP interface such as:

$$
\mathcal{P}(t)=4 \pi R^{2} g \Delta T(t)
$$

where $\Delta T$ is defined such as:

$$
\Delta T(t) \equiv T\left(R^{-}, t\right)-T\left(R^{+}, t\right)
$$

(in this work, the symbol $\equiv$ symbolizes a definition). The released heat power $\mathcal{P}(t)$ is also related to the temperature gradient on the NP surface through the energy conservation equation:

$$
\mathcal{P}(t)=-4 \pi R^{2} \kappa_{\mathrm{w}} \partial_{r} T(R, t) .
$$

Equations (3) and (5) yield a modification of the second boundary condition of system (2) at the nanoparticle interface $r=R$ :

$$
-\partial_{r} T\left(R^{+}, t\right)=\frac{1}{l_{\mathrm{K}}} \Delta T(t)
$$

where $l_{\mathrm{K}}=\kappa_{\mathrm{w}} / g$ is named the Kapitza length and $1 / g$ is the associated Kapitza resistivity.

Let us define from now two dimensionless constants that we shall often use in the following,

$$
\begin{aligned}
& \beta \equiv \frac{\rho_{\mathrm{w}} c_{\mathrm{w}}}{\rho_{\mathrm{Au}} c_{\mathrm{Au}}} \approx 1.680 \\
& \gamma \equiv \frac{\kappa_{\mathrm{Au}}}{\kappa_{\mathrm{w}}} \approx 512
\end{aligned}
$$

and one dimensionless parameter:

$$
\lambda_{\mathrm{K}} \equiv \frac{\kappa_{\mathrm{w}}}{g R}=\frac{l_{\mathrm{K}}}{R}
$$

that is the Kaptiza length normalized by the NP radius $R$. We also introduce dimensionless space $\rho$ and time $\tau$ variables defined such as:

$$
\begin{aligned}
\rho & \equiv r / R \\
\tau & \equiv a_{\mathrm{w}} t / R^{2}
\end{aligned}
$$

where $a=\kappa / \rho c$ is called the thermal diffusivity. $R$ and $R^{2} / a_{\mathrm{w}}$ are indeed the natural space and time units associated to the system. Within this work, we will make an extensive use of dimensionless variables and constants, first because it yields simpler, more natural formulae and more general results, and then because it shows how the algorithm can be properly written, i.e. working with numbers close to one and not unnecessary powers of ten. However, for the sake of simplicity, even when using normalized variables, we shall keep the same function names - e.g. $T(r, t)$ and $T(\rho, \tau)$. But this mathematical digression should not cause any clarity issue.

Using these dimensionless variables, parameters and constants, the set of equations (2) along with the additional boundary condition (6) read then:

$$
\left\{\begin{array}{l}
\text { Diffusion equations: } \\
\partial_{\tau} T(\rho, \tau)=\frac{\gamma \beta}{\rho^{2}} \partial_{\rho}\left(\rho^{2} \partial_{\rho} T(\rho, \tau)\right)+p(\rho, \tau) \text { for } \rho<1 \\
\partial_{\tau} T(\rho, \tau)=\frac{1}{\rho^{2}} \partial_{\rho}\left(\rho^{2} \partial_{\rho} T(\rho, \tau)\right) \text { for } \rho>1 \\
\text { Boundary conditions at } \rho=1 \text { : } \\
\partial_{\rho} T\left(1^{+}, \tau\right)=\gamma \partial_{\rho} T\left(1^{-}, \tau\right)=-\frac{1}{\lambda_{\mathrm{K}}} \Delta T(\tau)
\end{array}\right.
$$

where the Laplacian operator $\nabla^{2}$ has been reformulated using spherical coordinates.

From now on, the use of dimensionless formalism will not be systematic, but preferred when it simplifies the notations and make the results more general. 
TABLE I. Physical constants used in this work associated to gold and water. ${ }^{\mathrm{a}}$.

\begin{tabular}{lccc}
\hline \hline Name & Gold & Water & Unit \\
\hline Thermal conductivity $\kappa$ & 317 & 0.60 & $\mathrm{~W} / \mathrm{m} / \mathrm{K}$ \\
Specific heat capacity $^{\mathrm{b}} c$ & 129 & 4187 & $\mathrm{~J} / \mathrm{kg} / \mathrm{K}$ \\
Mass density $\rho$ & 19.32 & 1.00 & $\times 10^{3} \mathrm{~kg} / \mathrm{m}^{3}$ \\
Thermal diffusivity $a$ & 127 & 0.143 & $\times 10^{-6} \mathrm{~m}^{2} / \mathrm{s}$ \\
\hline \hline
\end{tabular}

${ }^{\text {a }}$ Values at approx. $25^{\circ} \mathrm{C}$ taken from ref. [42]

$\mathrm{b}$ at constant pressure

\section{B. Numerical method}

The set of equations (12) has no simple analytical solution. We chose to solve it numerically by developing a finite difference method (FDM) and a Runge-Kutta (RK) algorithm. ${ }^{43}$ Basically, it is based on a spatio-temporal discretization of the system of equations (12) according to:

$$
\begin{aligned}
\rho_{i} & \equiv i \times \delta \rho & i \in[0, N] \\
\tau_{j} & \equiv j \times \delta \tau & j \in[0, M] \\
T_{i, j} & \equiv T\left(\rho_{i}, \tau_{j}\right) & \\
\partial_{\rho} T(\rho, \tau) & \rightarrow \frac{T_{i+1, j}-T_{i, j}}{\delta \rho} & \\
\partial_{\tau} T(\rho, \tau) & \rightarrow \frac{T_{i, j+1}-T_{i, j}}{\delta \tau} . &
\end{aligned}
$$

This discretization procedure is associated with a RK algorithm of the fourth order (RK4) that ensures a higher accuracy - compared to regular Euler algorithms of the first order - in the estimation of $T_{i, j}$ at each spatiotemporal step.

Further details regarding the numerical algorithm are given in the appendix.

\section{C. cw illumination}

Before studying what occurs under femtosecond (fs) pulsed illumination, it is worth describing first what happens under continuous (cw) illumination. We consider in this paragraph a uniform cw illumination of irradiance $I$ and wavelength $\lambda_{0}$.

Under cw illumination, the establishment of the steady state temperature profile will be preceded by a transient evolution. By dimensional analysis of the two diffusion equations of system (2), one find that two time scales come into play:

$$
\begin{gathered}
\tau_{\mathrm{d}}^{\mathrm{w}}=\frac{\rho_{\mathrm{w}} c_{\mathrm{w}}}{\kappa_{\mathrm{w}}} R^{2}=\frac{R^{2}}{a_{\mathrm{w}}}, \\
\tau_{\mathrm{d}}^{\mathrm{Au}}=\frac{\rho_{\mathrm{Au}} c_{\mathrm{Au}}}{\kappa_{\mathrm{Au}}} R^{2}=\frac{R^{2}}{a_{\mathrm{Au}}} .
\end{gathered}
$$

$\tau_{\mathrm{d}}^{\mathrm{w}}$ is the characteristic time associated to the evolution of the temperature profile in the surrounding water while $\tau_{\mathrm{d}}^{\mathrm{Au}}$ characterizes the temperature evolution inside the gold NP. Since $a_{\mathrm{Au}} \gg a_{\mathrm{w}}$, the thermalization inside the NP occurs much faster. Consequently, one can consider that the global establishment of the temperature profile of the overall system is governed by the time scale $\tau_{d}^{\mathrm{w}}$.

We consider now the final steady state regime. The set of equations reads now:

$$
\begin{cases}\kappa_{\mathrm{Au}} \nabla^{2} T(r)=-p(r) & \text { for } r<R \\ \kappa_{\mathrm{w}} \nabla^{2} T(r)=0 & \text { for } r>R \\ \kappa_{\mathrm{w}} \partial_{r} T\left(R^{+}\right)=\kappa_{\mathrm{Au}} \partial_{r} T\left(R^{-}\right) \\ =-g \Delta T\end{cases}
$$

where $\Delta T \equiv T\left(R^{-}\right)-T\left(R^{+}\right)$. If one consider an average power density $p_{0}=\mathcal{P}_{0} / V$, the solution has a simple form and reads:

$$
\begin{aligned}
& T^{\mathrm{cw}}(r)=\frac{\mathcal{P}_{0}}{4 \pi \kappa_{\mathrm{w}} r} \quad \text { for } r>R \\
& T^{\mathrm{cw}}(r)=\frac{\mathcal{P}_{0}}{4 \pi \kappa_{\mathrm{w}} R}\left[1+\frac{1}{2 \gamma}\left(1-\frac{r^{2}}{R^{2}}\right)+\lambda_{\mathrm{K}}\right] \quad \text { for } r<R
\end{aligned}
$$

where $\mathcal{P}_{0}$ is the heat power dissipated in the NP. Note that the temperature profile outside the NP does not depend on the NP surface conductivity. ${ }^{44}$ Since $\gamma \gg 1$, one can usually consider - whatever the NP size - that the inner temperature of the NP is uniform and equals:

$$
T_{\mathrm{NP}}^{\mathrm{cw}}=\frac{\mathcal{P}_{0}}{4 \pi \kappa_{\mathrm{w}} R}\left(1+\lambda_{\mathrm{K}}\right)=\frac{\sigma_{\mathrm{abs}} I}{4 \pi \kappa_{\mathrm{w}} R}\left(1+\lambda_{\mathrm{K}}\right)
$$

where $\sigma_{\text {abs }}$ is the optical absorption cross section of the NP. ${ }^{2,45}$ For spherical nanoparticle smaller than $2 R \sim 30$ $\mathrm{nm}$, a good approximation can be used: ${ }^{46}$

$$
\text { where } \begin{aligned}
\sigma_{\mathrm{abs}} & =k \operatorname{Im}(\alpha)-\frac{k^{4}}{6 \pi}|\alpha|^{2} \\
& =\frac{\alpha_{0}}{1-(2 / 3) i k^{3} \alpha_{0}} \\
\text { and } \quad \alpha_{0} & =4 \pi R^{3} \frac{\varepsilon_{\mathrm{Au}}-\varepsilon_{\mathrm{w}}}{\varepsilon_{\mathrm{Au}}+2 \varepsilon_{\mathrm{w}}}
\end{aligned}
$$

with $\varepsilon_{\mathrm{Au}}$ the gold permittivity and $\varepsilon_{\mathrm{w}}$ the water permittivity.

From equation (19), we obtain thus a simple and very useful formula that gives the steady-state temperature of small spherical NP $(R<15 \mathrm{~nm})$ under cw illumination:

$$
\begin{aligned}
& T_{\mathrm{NP}}^{\mathrm{cw}}=\frac{k I R^{2}}{\kappa_{\mathrm{w}}} \operatorname{Im}\left(\frac{\varepsilon_{\mathrm{Au}}-\varepsilon_{\mathrm{w}}}{\varepsilon_{\mathrm{Au}}+2 \varepsilon_{\mathrm{w}}}\right), \\
& T_{\mathrm{NP}}^{\mathrm{cw}} \approx 2.00 \times \frac{k I R^{2}}{\kappa_{\mathrm{w}}} .
\end{aligned}
$$

The latter formula applies for $\lambda_{0}=520 \mathrm{~nm}$. No interface resistivity is assumed in this formula $\left(\lambda_{\mathrm{K}}=0\right)$. If the $\mathrm{NP}$ is not necessarily smaller than $2 R=30 \mathrm{~nm}$, the previous formalism becomes inappropriate and the more sophisticated and general Mie theory has to be used. ${ }^{46}$ Within this model, the absorption cross section reads:

$$
\sigma_{\mathrm{abs}}=\frac{2 \pi}{k^{2}} \sum_{j=1}^{\infty}(2 j+1)\left(\left|a_{j}\right|^{2}+\left|b_{j}\right|^{2}\right)
$$

where

$$
\begin{aligned}
& a_{j}=\frac{m \psi_{j}(v) \psi_{j}^{\prime}(u)-\psi_{j}(u) \psi_{j}^{\prime}(v)}{m \psi_{j}(v) \xi_{j}^{\prime}(u)-\xi_{j}(u) \psi_{j}^{\prime}(v)}, \\
& b_{j}=\frac{\psi_{j}(v) \psi_{j}^{\prime}(u)-m \psi_{j}(u) \psi_{j}^{\prime}(v)}{\psi_{j}(v) \xi_{j}^{\prime}(u)-m \xi_{j}(u) \psi_{j}^{\prime}(v)}
\end{aligned}
$$


and $m^{2}=\varepsilon_{\mathrm{Au}} / \varepsilon_{\mathrm{w}}, u=k R$ and $v=m u$. The primes indicates differentiation with respect to the argument in parenthesis. $\psi_{j}$ and $\xi_{j}$ are Ricatti-Bessel functions defined such as:

$$
\begin{aligned}
& \psi_{j}(x)=\sqrt{\frac{\pi x}{2}} J_{j+\frac{1}{2}}(x), \\
& \xi_{j}(x)=\sqrt{\frac{\pi x}{2}}\left[J_{j+\frac{1}{2}}(x)+i Y_{j+\frac{1}{2}}(x)\right] .
\end{aligned}
$$

$J_{\nu}$ and $Y_{\nu}$ are the Bessel functions of first and second order respectively. The derivatives can be expressed as follows:

$$
\begin{aligned}
& \psi_{j}^{\prime}(x)=\psi_{j-1}(x)-\frac{j}{x} \psi_{j}(x), \\
& \xi_{j}^{\prime}(x)=\xi_{j-1}(x)-\frac{j}{x} \xi_{j}(x) .
\end{aligned}
$$

In the following, the Mie theory will be used whenever a calculation of the absorption cross section is required.

\section{Pulsed illumination \& initial temperature increase}

We consider now a fs-pulsed illumination of average irradiance $\langle I\rangle$, pulsation rate $f$ and wavelength $\lambda_{0}$.

The absorption of a fs-pulse by a metal nanoparticle can be described as a three-step process ${ }^{47,48}$ that involves different time scales:

Electronic absorption. During the interaction with the fs-pulse, part of the incident pulse energy is absorbed by the gas of free electrons of the NP, much lighter and reactive than the ions of the lattice. The electronic gas thermalizes very fast to a Fermi-Dirac distribution over a time scale $\tau_{\mathrm{e}} \sim 100$ fs. ${ }^{48}$ This leads to a state of nonequilibrium within the NP: the electronic temperature $T_{\mathrm{e}}$ of the electronic gas has increased while the temperature of the lattice (phonons) $T_{\mathrm{p}}$ remains unchanged. The absorbed energy $\mathcal{E}_{0}$ reads:

$$
\mathcal{E}_{0}=\sigma_{\text {abs }}\langle I\rangle / f=\mathcal{P}_{0} / f .
$$

Electrons-phonons thermalization. Subsequently this hot electronic gas relaxes (cools down), through internal electron-phonon interaction characterized by a time scale $\tau_{\mathrm{e}-\mathrm{ph}}$ to thermalize with the ions of the gold lattice. This time scale is not dependent on the size of the NP except for NP smaller than $5 \mathrm{~nm}$ due to confinement effects. ${ }^{49}$ Above this size and for moderate pulse energy, the time scale is constant and equals $\tau_{\mathrm{e}-\mathrm{ph}} \sim 1.7$ ps. ${ }^{50-52}$ At this point, the NP is in internal equilibrium at a uniform temperature $\left(T_{\mathrm{e}}=T_{\mathrm{p}}\right)$, but is not in equilibrium with the surrounding medium that is still at the initial ambient temperature.

External heat diffusion. The energy diffusion to the surroundings usually occurs at higher characteristic time scale $\tau_{\mathrm{d}}$, which leads to a cooling of the NP and a heating of the surrounding liquid. The time scale of this process depends on the size of the NP and ranges from 100 ps to a few ns. For small NP $(<20 \mathrm{~nm})$, this third step can overlap in time with the electron-phonon thermalization $^{19}$ (as discussed hereafter).
If one consider that the electron-phonon thermalization (step 2) occurs much faster than the external heat diffusion (step 3), the NP temperature reaches an initial maximum temperature $T_{\mathrm{NP}}^{0}$ that is straightforward to estimate by energy consideration. It is related to the absorbed energy through the relation:

$$
\mathcal{E}_{0}=V \rho_{\mathrm{Au}} c_{\mathrm{Au}} T_{\mathrm{NP}}^{0}
$$

where $V$ is the volume of the NP and $V \rho_{\mathrm{Au}} c_{\mathrm{Au}}$ its heat capacity. Using equation (25), we find that the maximum initial NP temperature is:

$$
T_{\mathrm{NP}}^{0}=\frac{\sigma_{\mathrm{abs}}\langle I\rangle}{V \rho_{\mathrm{Au}} c_{\mathrm{Au}} f} .
$$

This formula is not restricted to spherical nanoparticles. As an example, for a gold nanorod, $50 \mathrm{~nm}$ long and 12 $\mathrm{nm}$ in diameter, at the plasmonic resonance $\left(\lambda_{0}=800\right.$ $\mathrm{nm}$ ), considering a random orientation, $f=86 \mathrm{MHz}$ and $\langle I\rangle=1.0 \mathrm{~mW} / \mathrm{\mu m}^{2}$, we obtain $T_{\mathrm{NP}}^{0} \approx 30^{\circ} \mathrm{C}$. Note that for a given laser power, the temperature increase does not depend on the pulse duration, but only on the pulse energy $\langle I\rangle / f$.

It is worth comparing the instantaneous temperature increase $T_{\mathrm{NP}}^{0}$ after a single fs-pulse and the steady-state temperature $T_{\mathrm{NP}}^{\mathrm{cw}}$ achieved under $\mathrm{cw}$ illumination. From Eqs. (19) and (27), we obtain a dimensionless number $\eta_{0}$ that quantifies the gain obtained when using pulsed illumination:

$$
\eta_{0} \equiv \frac{T_{\mathrm{NP}}^{0}}{T_{\mathrm{NP}}^{\mathrm{cw}}}=\frac{3 \beta a_{\mathrm{w}}}{f R^{2}\left(1+\lambda_{\mathrm{K}}\right)} .
$$

Figure 2 illustrates what $\eta_{0}, T_{\mathrm{NP}}^{0}$ and $T_{\mathrm{NP}}^{\mathrm{cw}}$ represent on an particular example. This useful formula is only valid for large values of $\eta_{0}$. When $R$ or $f$ tends to be high, successive pulses may overlap (as explained later in section II H), which makes the assumption of a single pulse illumination wrong. Furthermore, for very small particles, a temperature damping effect occurs (by a typical factor 2) and $T_{\mathrm{NP}}^{0}$ is not the initial temperature, as explained hereafter. Consequently, this formula is a good approximation of the temperature gain achieved under fs-pulsed illumination compared to $\mathrm{cw}$ illumination as far as $\eta_{0}$ remains large and the NP radius not too small $(R>10 \mathrm{~nm})$. The true values of $\eta_{0}-$ i.e. whatever the NP radius $R$ and surface conductivity $g$ and without approximations - is numerically computed and discussed in section II G.

\section{E. Subsequent evolution of the temperature profile}

We discuss now the subsequent evolution of the temperature field $T(r, t)$ after a single-pulse illumination, i.e. after an initial temperature increase $T_{\mathrm{NP}}^{0}$.

In the ideal case consisting of a point-like $\mathrm{NP}(R \rightarrow$ 0 ), the heat power density can be described by a Dirac distribution:

$$
\rho_{\mathrm{w}} c_{\mathrm{w}} \partial_{t} T(r, t)=\kappa_{\mathrm{w}} \nabla^{2} T(r, t)+\mathcal{E}_{0} \delta(\mathbf{r}) \delta(t) .
$$

This ideal problem has a simple analytical solution that reads:

$$
T(r, t)=\frac{\mathcal{E}_{0}}{c_{\mathrm{w}} \rho_{\mathrm{w}}} \frac{1}{\left(4 \pi a_{\mathrm{w}} t\right)^{3 / 2}} \exp \left(-\frac{r^{2}}{4 \pi a_{\mathrm{w}} t}\right) .
$$




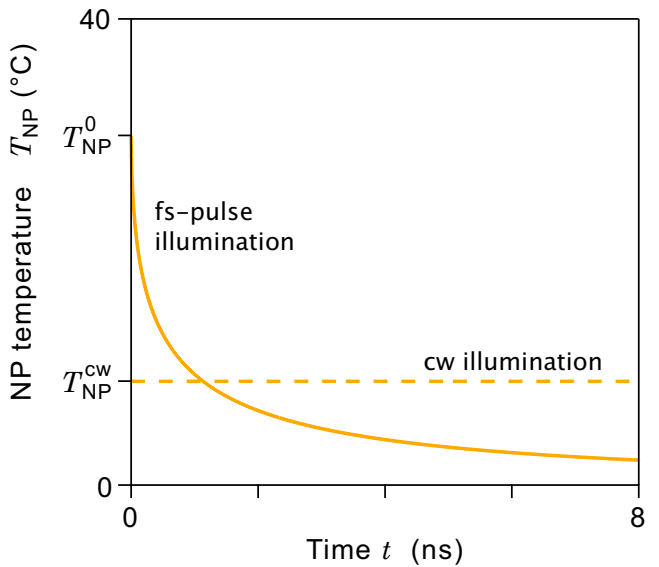

FIG. 2. Evolution of the temperature of a NP a radius $R=50$ $\mathrm{nm}$ under $\mathrm{cw}$ illumination (dashed line) and after a singlepulse illumination (solid line) for a given irradiance $\langle I\rangle=0.1$ $\mathrm{mW} / \mathrm{\mu m}^{2}$. This figure aims at defining visually the initial temperature increase $T_{\mathrm{NP}}^{0}$ under pulsed illumination and the steady state temperature $T_{\mathrm{NP}}^{\mathrm{cw}}$ under $\mathrm{cw}$ illumination. For this particular case, $T_{\mathrm{NP}}^{0}=31^{\circ} \mathrm{C}$ while $T_{\mathrm{NP}}^{\mathrm{cW}}=9.2^{\circ} \mathrm{C}$.

Then, the envelope $T_{\max }(r) \equiv \max _{t}(T(r, t))$ of the temperature profile over time can be easily obtained by using formula (30) and calculating the time $t$ for which $\partial_{t} T(r, t)=0$ for any position $r$. It yields a temperature envelope:

$$
T_{\max }(r)=\frac{1}{3 \sqrt{3}} \frac{\mathcal{E}_{0}}{c_{\mathrm{w}} \rho_{\mathrm{w}}} \frac{1}{r^{3}} .
$$

Interestingly, the temperature profile under pulsed illumination follows thus a $1 / r^{3}$ spatial decrease, which makes a real difference compared to continuous illumination characterized by a steady state profile in $1 / r$ (see Eq. (17)). This is a first clue that pulsed illumination achieves a much higher temperature confinement around the NP.

We consider now the more general and realistic case of a spherical NP defined by a finite radius $R \neq 0$. In this case, no simple analytical solution exists but some approximations can be done to simplify the problem. First, the initial temperature profile $T(r, 0)$ can be considered as uniform (equals $T_{\mathrm{NP}}^{0}$ ) inside the NP since the electronphonon thermalization usually occurs much faster than the external heat diffusion. Then, one can also suppose that the NP temperature remains uniform during the evolution of the system since $\kappa_{\mathrm{Au}} \gg \kappa_{\mathrm{w}}$. Hence,

$$
\forall t, \quad T(r, t)=T_{\mathrm{NP}}(t) \quad \text { for } r<R .
$$

The validity of these two approximations will be investigated and discussed in more details in section II G. Under these hypotheses, the system of equations (2) can be simplified:

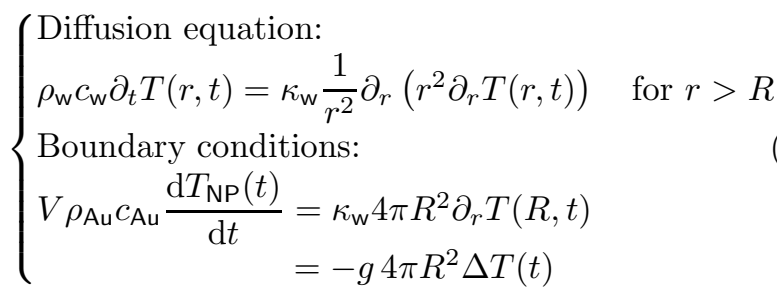

The first equation is the heat diffusion equation outside the NP. The two other equations come from considerations of energy conservation and will control the boundary condition at the NP interface $(r=R)$.

Interestingly, two new characteristic times arise from the boundary equations. They read:

$$
\begin{aligned}
\tau_{\mathrm{d}}^{\mathrm{NP}} & =R^{2} \frac{\rho_{\mathrm{Au}} c_{\mathrm{Au}}}{3 \kappa_{\mathrm{w}}}, \\
\tau_{\mathrm{d}}^{\mathrm{s}} & =\frac{R \rho_{\mathrm{Au}} c_{\mathrm{Au}}}{3 g} .
\end{aligned}
$$

These characteristic times are associated to the evolution of the nanoparticle average temperature - i.e. the heat energy stored in the NP. When the surface resistivity $1 / g$ is high (resp. small), the evolution is governed by $\tau_{d}^{\mathrm{s}}$ (resp. $\left.\tau_{d}^{\mathrm{NP}}\right)$. These new time scales differ from $\tau_{\mathrm{d}}^{\mathrm{Au}}$ and $\tau_{\mathrm{d}}^{\mathrm{w}}$ that were respectively the characteristic times associated to the establishment of the internal temperature equilibrium inside the NP and temperature diffusion outside the NP. If the surface conductivity $g$ is small enough, the evolution of the NP temperature is governed by $\tau_{\mathrm{d}}^{\mathrm{s}}$. If the surface resistivity is negligible, the evolution is governed by $\tau_{\mathrm{d}}^{\mathrm{NP}}$. The temperature evolution inside and outside the NP is thus governed by a subtile interplay between four time scales. Three of them are linked by the relation:

$$
\tau_{\mathrm{d}}^{\mathrm{w}}=\gamma \beta \tau_{\mathrm{d}}^{\mathrm{Au}}=3 \beta \tau_{\mathrm{d}}^{\mathrm{NP}},
$$

which yields:

$$
\tau_{\mathrm{d}}^{\mathrm{w}} \approx \tau_{\mathrm{d}}^{\mathrm{NP}} \gg \beta \tau_{\mathrm{d}}^{\mathrm{Au}} .
$$

Regarding the fourth time scale $\tau_{\mathrm{d}}^{\mathrm{s}}$, it can be dominant or negligible depending on the values of $R$ and $g$.

Using dimensionless time $\tau$ and space $\rho$ variables, the system of equations (32) can be recast into this simpler form:

$$
\left\{\begin{array}{l}
\text { Diffusion equation: } \\
\partial_{\tau} T(\rho, \tau)=\frac{1}{\rho^{2}} \partial_{\rho}\left(\rho^{2} \partial_{\rho} T(\rho, \tau)\right) \quad \text { for } \rho>1 \\
\text { Boundary conditions: } \\
\frac{\mathrm{d} T_{\mathrm{NP}}(\tau)}{\mathrm{d} \tau}=3 \beta \partial_{\rho} T(1, \tau)=-\frac{3 \beta}{\lambda_{\mathrm{K}}} \Delta T(\tau)
\end{array}\right.
$$

where $\Delta T(\tau)=T_{\mathrm{NP}}(\tau)-T(1, \tau)$.

Using the RK4 algorithm, system (37) was resolved numerically. The discretization parameters are $(\delta \rho, \delta \tau, N, M)=\left(15 \times 10^{-3}, 4 \times 10^{-6}, 400,10^{6}\right)$. The interface resistivity $1 / g$ was set to zero (non-zero values are discussed in the next section). The result is presented in Fig. 3 that displays the universal normalized evolution of the temperature of a NP. This profile applies for any particle size since it uses the normalized variables $(\rho, \tau)$ : 


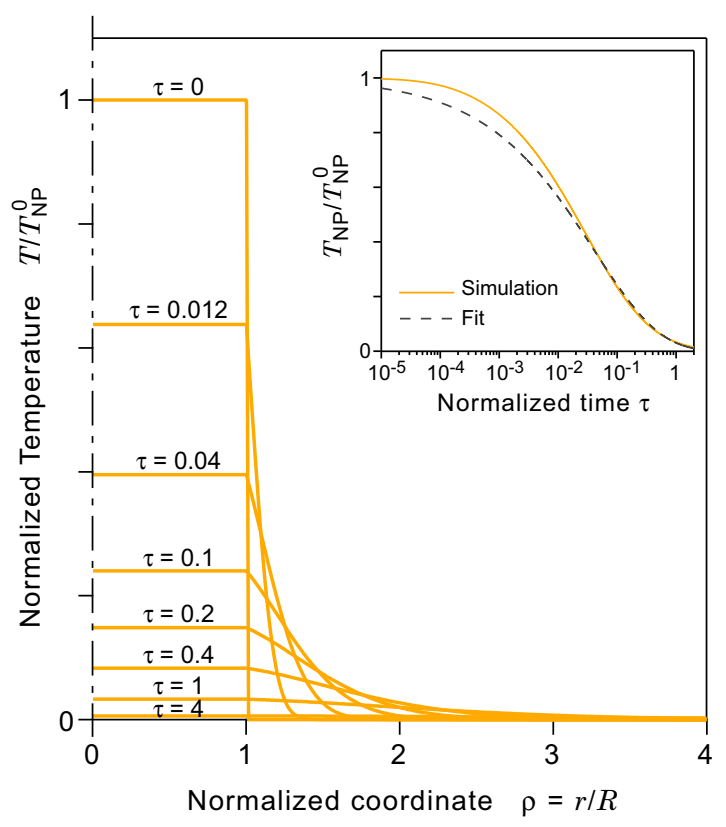

FIG. 3. Result of a numerical simulation showing the universal evolution of the temperature profile for a NP of radius $R$ at different normalized time $\tau=a_{\mathrm{w}} t / R^{2}$. No interface resistivity is considered $(1 / g=0)$. The inset shows the evolution of the NP inner temperature as function of time.

For a given particle size $R$, the normalized coordinate $\rho$ has to be multiplied by $R$ and the normalized time $\tau$ by $R^{2} / a_{\mathrm{w}}$ to recover the actual coordinate $r$ and time $t$. Hu and Hartland ${ }^{19}$ have shown experimentally that the NP temperature can be conveniently fitted using a stretched exponential function:

$$
F(\tau)=\mathrm{e}^{-\left(\tau / \tau_{0}\right)^{n}} .
$$

We used this function to fit the evolution of the NP temperature as represented in the inset of Fig. 3 (dashed line). The optimized fit parameters are $n=0.39$ and $\tau_{0}=0.041$. This yields a useful formula giving the normalized NP inner temperature evolution for any particle radius $R$ :

$$
F_{R}(t)=\exp \left[-\left(\frac{a_{\mathrm{w}} t}{0.041 R^{2}}\right)^{0.39}\right] .
$$

Note that this useful formula assumes a zero interface resistivity $1 / g$. For a finite value of $g$, the values of the fit parameters $\tau_{0}$ and $n$ are different and have to be recalculated using the FDM.

Figure 4 aims at comparing the temperature profiles under pulsed and $\mathrm{cw}$ illuminations. It replots the series of temperature profiles of Fig. 3 along with the temperature envelope and the temperature profile of the steady state (cw illumination) given by Eq. (17). As derived in the previous section, when considering a point-like $(R \rightarrow 0)$ source of heat, the envelope of the temperature profile follows a $1 / r^{3}$ profile outside the NP. When considering now a finite-size NP, it appears that such a simple law does not exist. Instead, a stretched exponential function can also be conveniently used to fit the
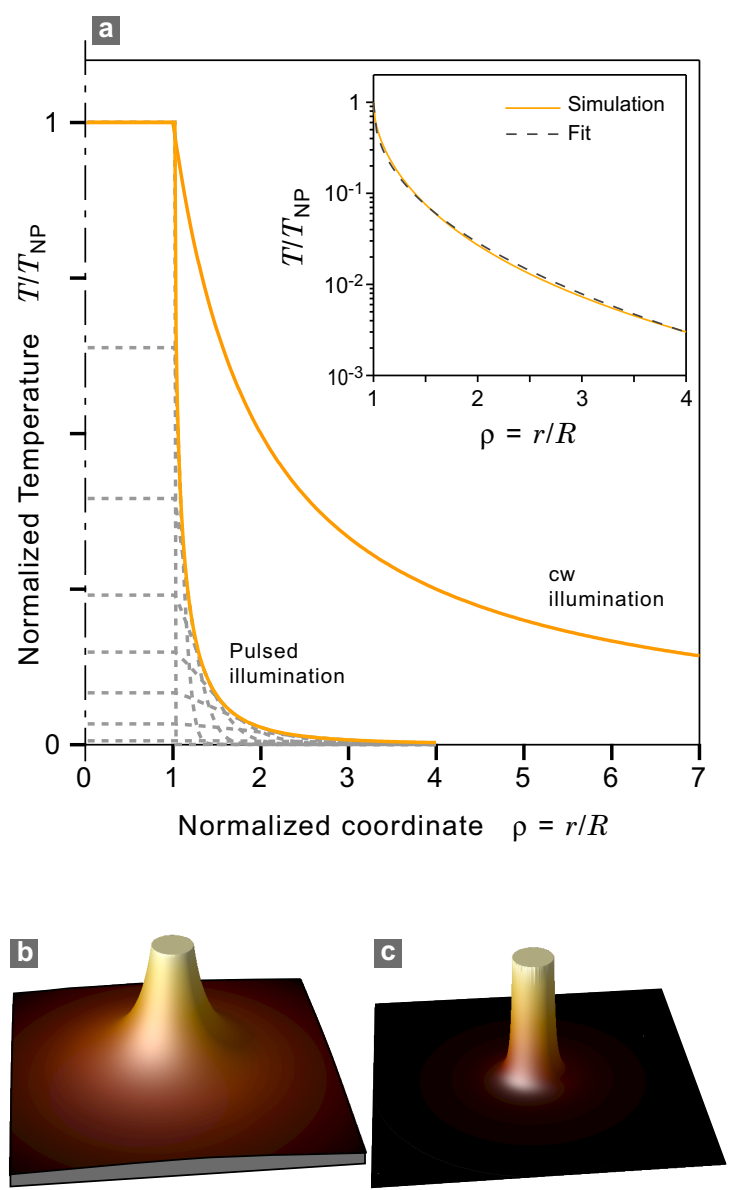

FIG. 4. a) Comparison between the steady state temperature profile under $\mathrm{cw}$ illumination and the envelope of the temperature evolution under pulsed illumination. The inset shows the fit of the NP temperature profile in the case of a pulsed illumination. b) Temperature profile around the NP under $\mathrm{cw}$ illumination. c) Envelope of the temperature profile over time around the NP during subsequent to a fs-pulse illumination.

envelope of the spatial temperature profile in the surrounding water:

$$
F(\rho)=\exp \left[-\left(\frac{\rho-1}{\rho_{0}}\right)^{n}\right]
$$

The fit parameters are $n=0.45$ and $\rho_{0}=0.060$. This results illustrate to what extent pulsed illumination achieves a much higher degree of temperature confinement compared to $\mathrm{cw}$ illumination.

Note that the evolution of the system is characterized by an energy conservation law. At any time $t$, the energy of the system is constant and reads:

$$
\mathcal{E}_{0}=\frac{4}{3} \pi R^{3} \rho_{\mathrm{Au}} c_{\mathrm{Au}} T_{\mathrm{NP}}(t)+\int_{R}^{\infty} \rho_{\mathrm{w}} c_{\mathrm{w}} 4 \pi r^{2} T(r, t) \mathrm{d} r
$$

or using the normalized variables and constants defined 
above, the normalized energy reads:

$$
\epsilon_{0}=\frac{T_{\mathrm{NP}}(\tau)}{T_{\mathrm{NP}}^{0}}+\int_{1}^{\infty} 3 \beta \rho^{2} \frac{T(\rho, \tau)}{T_{\mathrm{NP}}^{0}} \mathrm{~d} \rho=1 .
$$

This conservation law can be conveniently used in numerical simulations as a verification of the consistency of the result. For example, in the simulations shown in figure 3 , it varied by less than $0.2 \%$.

\section{F. Finite conductivity of the gold-water interface}

In this section, we shall go one step further into the refinement of the analytical description of the problem. We still consider the NP temperature as uniform, but we take into account a finite interface conductivity $g$. The set of equations describing the system is given by (37).

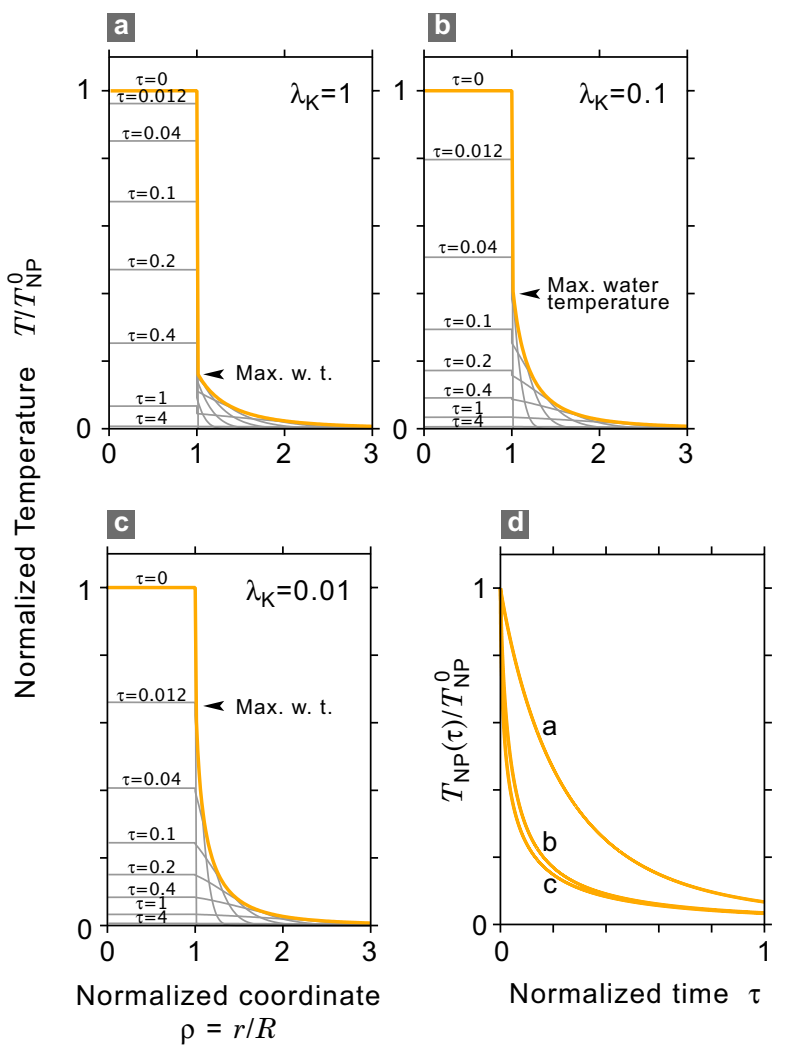

FIG. 5. a, b, c) Temperature envelope of the temperature evolution for three different values of normalized Kapitza length $\lambda_{\mathrm{K}}$. Temperature profiles are also represented at different times (in gray). d) Evolution of the NP inner temperature corresponding to the three previous situations.

Fig. 5 displays the evolution of the temperature profiles for a set of different normalized Kapitza length (i.e. gold/water interface conductivity). Usual values of the interface conductivity $g$ range from 50 to $\infty$ $\mathrm{MW} /\left(\mathrm{m}^{2} \mathrm{~K}\right) \cdot{ }^{38,41}$ As an example, gold nanorods coated with CTAB molecules are endowed with a typical surface conductivity of $130 \mathrm{MW} /\left(\mathrm{m}^{2} \mathrm{~K}\right) \cdot{ }^{38}$ For $\mathrm{NP}$ radii ranging from 5 to $50 \mathrm{~nm}$, typical normalized Kaptiza length $\lambda_{\mathrm{K}}$ are thus ranging from approximatively 0 to 2 .

For large Kapitza length (high interface resistivity), the heating of the surrounding fluid can be highly inefficient as observed in Fig. 5a. This is due to the fact that the high resistivity of the NP interface tends to slow down the heat release, which yield a weaker maximum temperature in water. The same amount of energy is released in the surroundings but more softly.

\section{G. Beyond the approximation of instantaneous temperature increase}

In the previous section, we used two approximations regarding the NP temperature to simplify the problem.

First, we have considered that the NP temperature increase $T_{\mathrm{NP}}^{0}$ subsequent to the pulse of light was instantaneous and uniform inside the NP. This was because the characteristic time of electron-phonon scattering $\left(\tau_{\mathrm{e}-\mathrm{ph}} \sim 1.7 \mathrm{ps}\right)$ is usually smaller than the diffusion time into the surrounding (see Eq. (33)), but this may become non-valid for small NP.

Second, we assumed that the NP temperature remained uniform inside the NP during the overall evolution. That was because the thermal conductivity of gold is much larger than the one of water.

Yet, the validity of these two approximations may depend a priori on the size $R$ and the interface conductivity $g$ of the NP. The numerical algorithm used in this work allows one to consider the inner temperature of the NP as not necessarily uniform and to investigate the step where the temperature increases while the NP is being illuminated by the pulse. We can thus investigate the validity of the two approximations mentioned above by simulating the evolution of the inner temperature profile, both during the electron-phonon thermalization and during the subsequent heat diffusion in the surrounding medium. This is the purpose of this section.

During the electron-phonon thermalization, one can assume that the heat power density $p(r, t)$ is uniform all over the NP.

$$
p(r, t)=p_{0}(t)=\frac{\mathcal{E}_{0}}{V \tau_{\mathrm{e}-\mathrm{ph}}} \exp \left(-t / \tau_{\mathrm{e}-\mathrm{ph}}\right) .
$$

Indeed, we have seen in section II D that the electronic thermalization (step 1) occurs much faster than the energy transfer from the electrons to the lattice (step2). The heating of the lattice is thus performed by a uniformly hot electronic gas. The dimensionless form reads:

$$
p_{0}(\tau)=T_{\mathrm{NP}}^{0} \frac{\tau_{\mathrm{p}}^{\mathrm{w}}}{\tau_{\mathrm{e}-\mathrm{ph}}} \exp \left(-\tau \tau_{\mathrm{p}}^{\mathrm{w}} / \tau_{\mathrm{e}-\mathrm{ph}}\right) .
$$

Note that under $\mathrm{cw}$ illumination, the heat generation density is on the contrary highly non-uniform within the NP. ${ }^{53}$

Within this new approach characterized by an initial zero temperature profile and a heat power density in the NP, the energy conservation law reads now, at any 

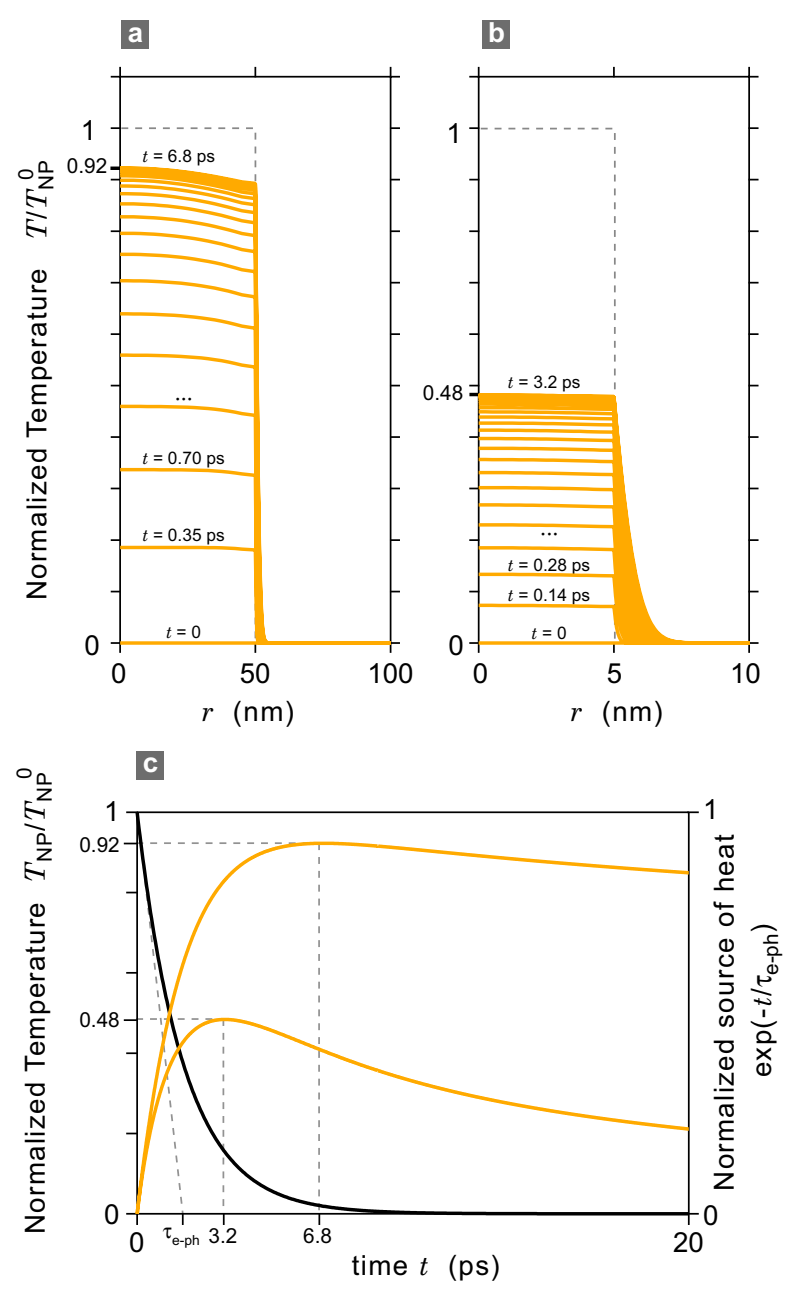

FIG. 6. Temperature rise subsequent to a femtosecond-pulsed illumination for a NP $100 \mathrm{~nm}$ in diameter (a) and $10 \mathrm{~nm}$ in diameter (b) (interface resistivity $1 / g=0$ ). The ideal temperature profile that the NP would achieve if there were no heat release in the surrounding is represented by a dashed line. c) Evolution of the temperature $T(r=0, t)$ for the two above-mentioned cases along with the normalized heat power $p_{0}(t)$ (dark line) delivered by the excited electronic gas to the phonons. The normalized temperature does not reach 1 due to heat release during the NP heating.

normalized time $\tau$, under $\mathrm{cw}$ illumination:

$$
3 \beta T_{\mathrm{NP}}^{\mathrm{cW}} \tau=\int_{0}^{1} 3 \rho^{2} T(\rho, \tau) \mathrm{d} \rho+\int_{1}^{\infty} 3 \rho^{2} \beta T(\rho, \tau) \mathrm{d} \rho
$$

and under pulsed illumination:

$$
\begin{aligned}
T_{\mathrm{NP}}^{0}\left[1-\exp \left(\frac{-\tau \tau_{\mathrm{d}}^{\mathrm{w}}}{\tau_{\mathrm{e}-\mathrm{ph}}}\right)\right] & =\int_{0}^{1} 3 \rho^{2} T(\rho, \tau) \mathrm{d} \rho \\
& +\int_{1}^{\infty} 3 \rho^{2} \beta T(\rho, \tau) \mathrm{d} \rho .
\end{aligned}
$$

Figure 6 presents the results of the numerical simulations for two NP sizes $R=5$ and $R=50 \mathrm{~nm}$, and for $1 / g=0$. It shows the temperature evolution during the heating phase from the initial zero uniform temperature up to the time when the NP temperature reaches its maximum value. We can see that when considering a possible heat leak into the surrounding during the pulse illumination, the NP temperature does not reach necessarily $T_{\mathrm{NP}}^{0}$. For large NP, the inner temperature profile suffers from some distortion when the temperature reaches its highest value (Fig. 6a). However, the maximum temperature reaches practically the ideal maximum temperature $T_{\mathrm{NP}}^{0}$. Indeed, one can see that the heat diffusion into the surroundings is almost absent. For small NP (Fig. 6b), the temperature diffusion into the surrounding medium during this initial step is much more visible. The direct consequence is that the NP temperature does not reach its maximum ideal value $T_{\mathrm{NP}}^{0}$ represented by dashed line in Fig. 6b. The evolution of the NP inner temperature $(T(r=0, t))$ for the two above mentioned cases $(R=5$ and $R=50 \mathrm{~nm}$ ) is plotted in Fig. 6c. The temperature profiles are displayed along with the evolution of the heat power $p_{0}(t)$ provided by the free electron gas. For small particles, it is clear that the time scale $\tau_{\mathrm{e}-\mathrm{ph}}$ becomes of the same magnitude as the characteristic time of the heat diffusion in the surrounding. This explains why the NP temperature cannot reach the maximum ideal temperature $T_{\mathrm{NP}}^{0}$. Note that the temperature $T_{\mathrm{NP}}^{0}$ used to normalized the curves in Fig. 6 depends on $R$ (cf. Eq. $(27))$ and is thus not the same for the two cases.

In any case, the temperature remains quasi-uniform inside the NP, which validate the usual assumption (31).

The maximum temperature achieved in the system is discussed in more details in Fig. 7. Temperatures are plotted as function of NP size $R$ and interface conductivity $g$. Figs. 7a-b represent the maximum temperature inside and outside the NP:

$$
\begin{aligned}
T_{\mathrm{NP}}^{\max } & =\max _{t}[T(r=0, t)], \\
T^{\max }\left(R^{+}\right) & =\max _{t}\left[T\left(r=R^{+}, t\right)\right]
\end{aligned}
$$

normalized by the ideal temperature increase $T_{\mathrm{NP}}^{0}$ (Eq. (27) and Fig. $7 \mathrm{~g}$ ). When $T_{\mathrm{NP}}^{\max } / T_{\mathrm{NP}}^{0} \ll 1$, it means that the heat release outside the NP is too fast. The characteristic time $\tau_{\mathrm{d}}^{\mathrm{w}}$ (Eq. (14)) becomes close to the characteristic time of the inner thermalization and step 2 and 3 occur almost simultaneously. This happens when the particle is too small and when the surface conductivity is not weak. When $T_{\mathrm{w}}^{\max } / T_{\mathrm{NP}}^{0} \ll 1$, it means that the water temperature increase is not optimum. This happens when the NP is too small, or when $g$ is too small, giving rise to a large temperature drop at the NP interface.

Figures $7 \mathrm{c}-\mathrm{d}$ represent the maximum temperature inside and outside the NP for a given irradiance $I=0.1$ $\mathrm{mW} / \mathrm{\mu m}^{2}$. Interestingly, we evidence an optimal $\mathrm{NP}$ size. NPs around $2 R=40 \mathrm{~nm}$ turn out to be the most efficient nano-source of heat for a given laser irradiance. Below this size, the cooling of the NP is too fast and the temperature has no time to reach $T_{\mathrm{NP}}^{0}$, as explained above. Above this size, the absorption cross section is no longer proportional to the volume of the nanoparticle, as seen in Fig. $7 \mathrm{f}$ and in Eq. (20), which tends to damp the temperature increase. This trend is also observed in Fig. $7 \mathrm{~d}$ that plots the associated temperature increase at the vicinity of the NP under the same illumination conditions. Moreover, we can see that to achieve a high 

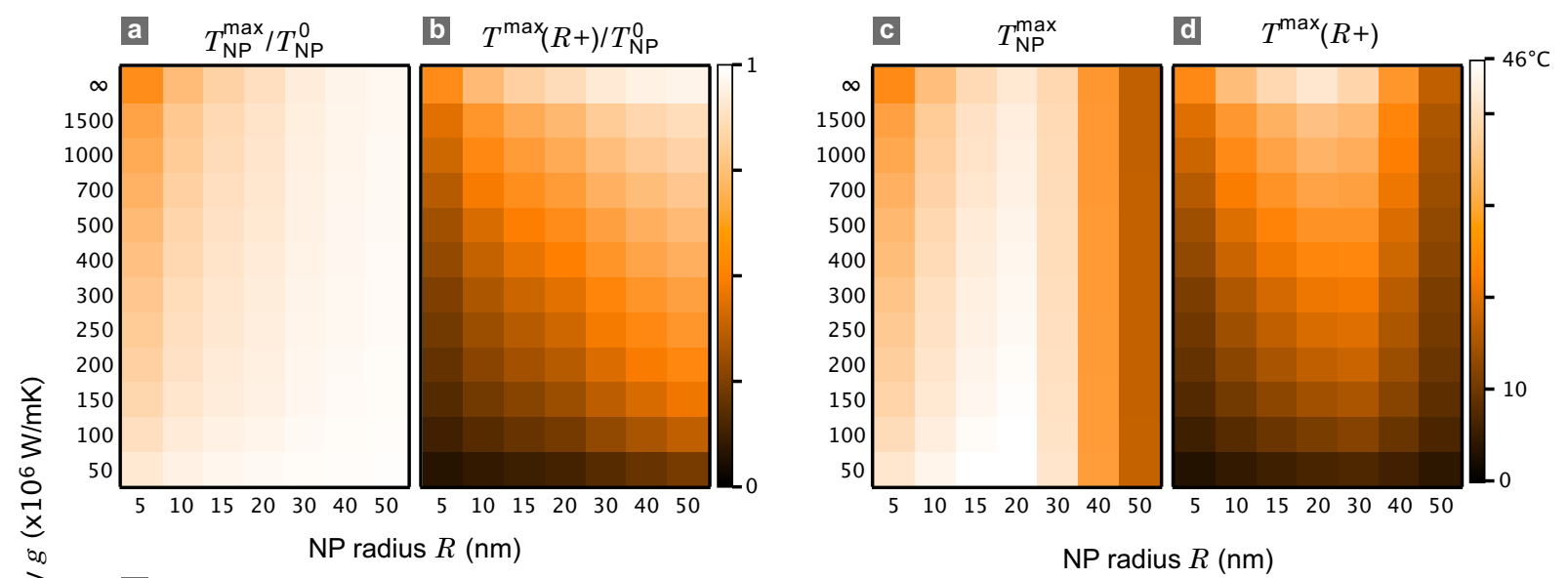

\section{e}
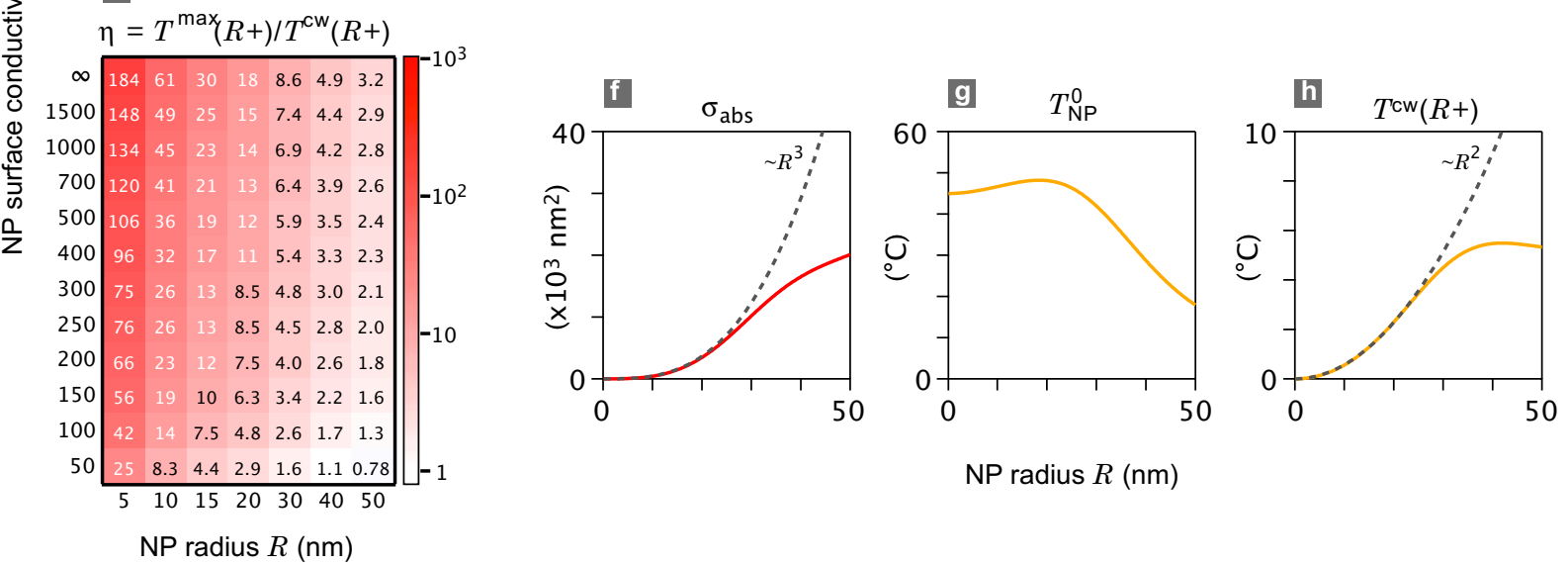

FIG. 7. a) Numerical calculations of the temperature maximum of the NP normalized by the ideal temperature increase $T_{\mathrm{NP}}^{0}$ for a set of different NP radii and surface conductivities. b)Numerical calculations of the temperature maximum achieved in the surrounding medium normalized by the ideal temperature increase $\left.T_{\mathrm{NP}}^{0} . \mathrm{c}\right) \mathrm{NP}$ maximum temperature when illuminated by a pulsed laser, at a pulsation rate $f=86 \mathrm{MHz}$ and an irradiance $I=0.1 \mathrm{~mW} / \mathrm{\mu m}^{2}$. d) Maximum temperature of the surrounding medium under the same illumination conditions. e) Ratio between the maximum temperature on the NP surface achieved under pulsed illumination and the temperature on the NP surface under cw illumination (Eq. (17)). f) Absorption cross section of a spherical gold NP as function of radius obtained from Mie theory (Eq. (25)) (solid line). The deviation from the $R^{3}$ law is represented by a dashed line. g) Ideal temperature increase $T_{\mathrm{NP}}^{0}$ as function of NP radius. h) Temperature on the NP surface under cw illumination (calculated from Eq. (17)). The deviation from the $R^{2}$ law (see Eq. (24)) is represented by a dashed line.

temperature increase inside (resp. outside) the NP, a low (resp. high) surface conductivity $g$ is preferred.

Figure 7e aims at comparing the efficiency of pulsed versus $\mathrm{cw}$ illumination. In Eq. (2), we defined the gain $\eta_{0}$ with the approximation of an instantaneous temperature increase and no interface resistivity. Now, we can define the exact gain $\eta$ that takes into account the initial temperature damping and a finite interface conductivity:

$$
\eta=T^{\max }\left(R^{+}\right) / T^{\mathrm{cw}}\left(R^{+}\right) .
$$

We observe that for small NP and high surface conductivity (upper left corner of Fig. 7e), a fs-pulsed illumination achieves a temperature rise two orders of magnitude higher than $\mathrm{cw}$ illumination of the same average irradiance. However, depending on the NP size and surface conductivity, a pulsed illumination does not necessarily achieve a more pronounced temperature increase in the surrounding medium. Namely, for big NP around $2 R=100 \mathrm{~nm}$ in diameter, it appears that the use of pulsed illumination becomes inefficient since the gain in temperature reach a maximum value of around 3. For low surface resistivity, the surrounding maximum temperature can even be higher when using $\mathrm{cw}$ illumination (lower right corner of Fig. 7e). Consequently, the use of a fs-pulsed laser is not necessarily efficient if one want to achieve the highest temperature possible for a given laser power. This result questions the utility of fs-pulse illumination on lithographic structure, since usually bigger than $100 \mathrm{~nm}$.

\section{H. Influence of the pulse repetition rate}

When the relaxation time of the system is sufficiently long, the temperature of the NP has no time to return to zero between two successive pulses. This effect has not been considered so far and is the purpose of this section. The parameter that controls the appearance of such a 


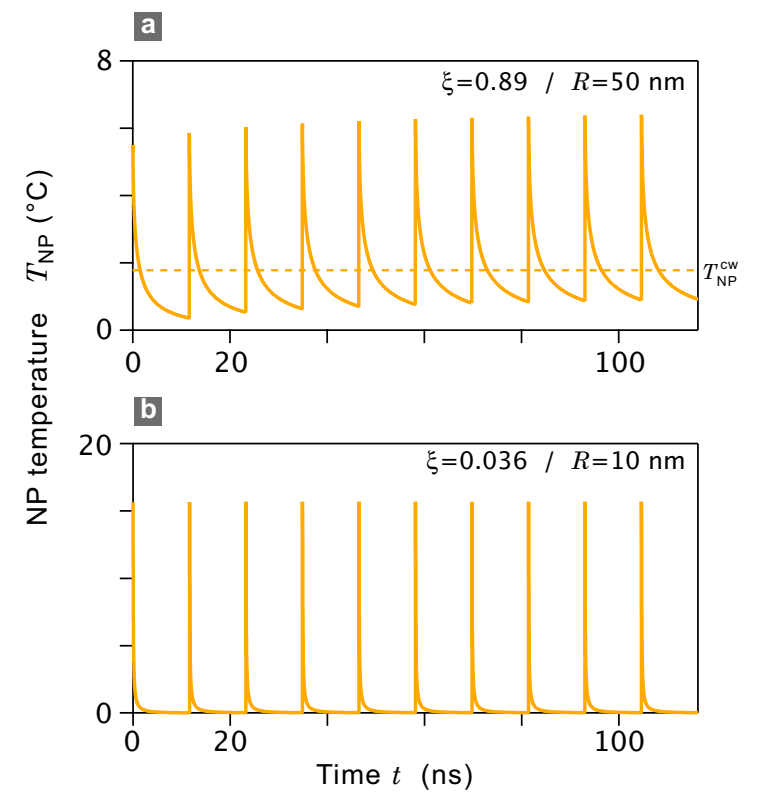

FIG. 8. NP temperature during a series of 10 pulses, $f=86$ $\mathrm{MHz},\langle I\rangle=1 \mathrm{~mW} / \mu^{2}$, for a NP radius $R=50 \mathrm{~nm}$ (a) and $R=10 \mathrm{~nm}$ (b). In figure a, the NP temperature corresponding to a cw illumination is $T_{\mathrm{NP}}^{\mathrm{cw}}=1.78^{\circ} \mathrm{C}$ and is represented by a dashed line. In the second case, the NP temperature under $\mathrm{cw}$ illumination is $T_{\mathrm{NP}}^{\mathrm{cW}}=0.30^{\circ} \mathrm{C}$ (not represented).

regime is:

$$
\xi \equiv f \tau_{\mathrm{d}}=f\left(\tau_{\mathrm{d}}^{\mathrm{NP}}+\tau_{\mathrm{d}}^{\mathrm{s}}\right)=f R^{2} \frac{\rho_{\mathrm{Au}} c_{\mathrm{Au}}}{\kappa_{\mathrm{w}}}\left(1+\lambda_{\mathrm{K}}\right) .
$$

Let $G_{R, g}(t)$ be the NP temperature evolution after a single-pulse illumination for a NP of radius $R$ and interface conductivity $g$. Note that a fit formula of $G_{R, \infty}(t)$ for $g \rightarrow \infty$, assuming a square initial temperature profile, is given by Eq. (39). Due to the linearity of all the equations governing the heat release and diffusion in the system, the NP temperature at any time $t$ after a series of $\mathrm{N}$ pulses at the repetition rate $f$ is:

$$
T_{\mathrm{NP}}(t)=\sum_{j=0}^{N-1} G_{R, g}(t-j / f) .
$$

Figure 8 plots the results of numerical simulations for two cases corresponding to two different NP radii. The interface resistivity $1 / g$ is assumed to equal zero. For a radius of $R=50 \mathrm{~nm}$ (Fig. 8a), $\xi$ is close to unity, which yields a temperature offset: the NP temperature has no time to return to zero between two successive pulses. For even larger NP, the temperature evolution would be even more smoothered and would tend to $T_{\mathrm{NP}}^{\mathrm{cw}}$. For weaker values of $\xi$, as shown in Fig. 8b, the successive temperature pulses do not overlap and a regime of time localization can be achieved.

\section{Extension to non-spherical nanoparticles}

The numerical technique we have developed could be extended to $2 \mathrm{D}$ systems modeling a NP with axial symmetry, like nanorods. It would require a $2 \mathrm{D}$ mesh and longer computation times. However, most of the results presented in this work can be easily extended to nonspherical NP without carrying out more sophisticated calculations: While the optical properties of NP particles depend very sensitively on the NP geometry - in particular the resonance frequency-, thermal processes of NP are only slightly dependent on the geometry. ${ }^{2}$ For non-spherical NP, we can define an effective radius $R_{\text {eff }}$ such that the NP volume equals:

$$
V=\frac{4}{3} \pi R_{\text {eff }}^{3} .
$$

Most of the reasonings we have done are also valid when replacing $R$ by this effective radius $R_{\text {eff }} .^{2}$ In particular, the equation (27) giving the ideal temperature increase $T_{\mathrm{NP}}^{0}$ remains exact whatever the shape of the NP us-

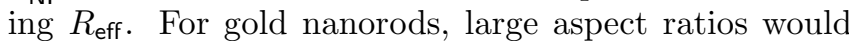
tend to make the cooling of the NP slightly faster by increasing the surface to volume ratio. However, all the orders of magnitude of time and space will remain identical as long as the aspect ratio remains moderate (less than $\sim 4$ ).

\section{SUMMARY AND CONCLUSION}

To summarize, we have developed a versatile and yet simple numerical framework to investigate femtosecondpulsed optical heating of spherical gold nanoparticles (NPs). This approach enabled us to address different models, from simple to more sophisticated, and to discuss the validity of their approximations. Most of the reasonings and calculations are made using dimensionless variables, parameters and constants, which enabled us to compute universal behaviors. The constitutive equations governing the system are derived and explained. We show how such a physical system turns out to be governed by a subtile interplay between four characteristic time scales.

We investigated the influence of the size of the NP: For small NP (diameter $<30 \mathrm{~nm}$ ), the temperature rise is not as high as expected due to fast heat release. For bigger NP, the temperature rise is damped because large NP are not efficient absorbers. This leads to a particle size compromise around $40 \mathrm{~nm}$ that optimizes the temperature increase (for a given laser irradiance).

The role of a possible molecular coating is also investigated in details based on considerations on interface thermal resistivity. For high interface resistivity, a temperature damping is observed outside the NP while an enhanced temperature increase is observed inside. A high interface resistivity tends in parallel to slow down the heat release in the surrounding medium making the heating of the surroundings less efficient.

A detailed comparison between $\mathrm{cw}$ and pulsed illumination was drawn and two main results came out: i) While a temperature profile in $1 / r$ in the surrounding medium is observed under $\mathrm{cw}$ illumination, a much more confined temperature envelope in $1 / r^{3}$ characterizes a pulsed illumination. A refined model even further demonstrates a higher degree of confinement with a spatial profile following a stretched exponential. ii) Unexpectedly, pulsed illumination does not necessarily 
achieve much higher temperature increase in the surroundings compared to $\mathrm{cw}$, especially for nanoparticles bigger than $100 \mathrm{~nm}$ (typically lithographic plasmonic structures). It can even be worse when the gold particle is endowed with a poor thermal surface conductivity (due to an hydrophobic molecular coating for example).

Finally, the influence of the repetition rate is discussed and two regimes are identified depending on the NP radius $R$ and the pulsation rate $f$ : One time-localization regime, where the temperature increase is confined spatially and temporally and one regime that tends to resemble to the regime observed under $\mathrm{cw}$ illumination.

Within this work, we restricted ourselves to gold nanoparticles with spherical geometry (radius $R$ ), but most of the results obtained herein are also valid for non- spherical particles when considered as particles of characteristic size $R$. The numerical techniques we developed could also be refined to address problems with $2 \mathrm{D}$ symmetries requiring a longer computation time. This versatile numerical technique could also take into account other materials than gold and water and various pulse durations, from femto- to nanosecond-scales.

\section{ACKNOWLEDGMENTS}

We thank Christian Girard, Philippe Réfrégier, Damien Riedel and Jérôme Wenger for helpful discussions.
* guillaume.baffou@fresnel.fr

1 A. O. Govorov and H. H. Richardson, Nano Today 2, 30 (2007).

2 G. Baffou, R. Quidant, and F. J. García de Abajo, ACS nano 4, 709 (2010).

3 A. Nitzan and L. E. Brus, J. Chem. Phys. 75, 2205 (1981).

${ }^{4}$ W. A. Challener, C. Peng, A. V. Itagi, D. Karns, W. Peng, Y. Peng, X. M. Yang, X. Zhu, N. J. Gokemeijer, Y.-T. Hsia, G. Ju, R. E. Rottmayer, M. A. Seigler, and E. C. Gage, Nat. Photon. 3, 220 (2009).

${ }^{5}$ L. Cao, D. Barsic, A. Guichard, and M. Brongersma, Nano Lett. 7, 3523 (2007).

${ }^{6}$ D. Pissuwan, S. M. Valenzuela, and M. B. Cortie, Trends Biotechnol. 24, 62 (2006).

7 P. K. Jain, I. H. El-Sayed, and M. A. El-Sayed, Nano Today 2, 18 (2007).

8 S. Lal, S. E. Clare, and N. J. Halas, Acc. Chem. Res. 41, 1842 (2009).

9 G. Han, P. Ghosh, M. De, and V. M. Rotello, NanoBioTechnology 3, 40 (2007).

10 A. G. Skirtach, C. Dejugnat, D. Braun, A. S. Susha, A. L. Rogach, W. J. Parak, H. Möhwald, and G. B. Sukhorukov, Nano Lett. 5, 1371 (2005).

11 L. Tong, Q. Wei, A. Wei, and J. X. Cheng, Photochemistry and photobiology 85, 21 (2009).

12 J. Butet, J. Duboisset, G. Bachelier, I. Russier-Antoine, E. Benichou, C. Jonin, and P. F. Brevet, Nano Lett. 10, 1717 (2010).

13 W. Lu, Q. Huang, G. Ku, X. Wen, M. Zhou, D. Guzatov, P. Brecht, R. Su, A. Oraevsky, L. V. Wang, and C. Li, Biomaterials 31, 2617 (2010).

14 S. Mallidi, T. Larson, J. Aaron, K. Sokolov, and S. Emelianov, Opt. Express 15, 6583 (2007).

15 A. Vogel and V. Venugopalan, Chem. Rev. 103, 577 (2003).

16 R. R. Anderson and J. A. Parrish, Science 220, 524 (1983).

17 V. K. Pustovalov, Chem. Phys. 308, 103 (2005).

18 A. N. Volkov, C. Sevilla, and L. V. Zhigilei, Appl. Surf. Sci. 253, 6394 (2007).

19 M. Hu and G. V. Hartland, J. Phys. Chem. B 106, 7029 (2002).

20 M. Hu, X. Wang, G. V. Hartland, P. Mulvaney, J. P. Juste, and J. E. Sader, J. Am. Chem. Soc. 125, 14925 (2003).

21 A. L. Tchebotareva, P. V. Ruijgrok, and M. Orrit, Laser Photonics Rev. 4, 581 (2010).

22 N. Large, L. Saviot, J. Margueritat, J. Gonzalo, C. N. Afonso, A. Arbouet, P. Langot, A. Mlayah, and J. Aizpurua, Nano Lett. 9, 3732 (2009).
${ }^{23}$ P. Chakravarty, W. Qian, M. A. El-Sayed, and M. R. Prausnitz, Nature Nanotech. 5, 607 (2010).

24 A. Vogel, J. Noack, G. Hüttman, and G. Paltauf, Appl. Phys. B 81, 1015 (2005).

25 D. Lapotko, Opt. Express 17, 2538 (2009).

26 E. Lukianova-Hleb, L. Hu, Y. andLatterini, L. Tarpani, S. Lee, R. A. Drezek, J. H. Hafner, and D. O. Lapotko, ACS nano 4, 2109 (2010).

27 A. Vogel, N. Linz, S. Freidank, and G. Paltauf, Phys. Rev. Lett. 100, 038102 (2008).

${ }^{28}$ V. Kotaidis, C. Dahmen, G. von Plessen, F. Springer, and A. Plech, J. Chem. Phys. 124, 184702 (2006).

29 S. Link, C. Burda, B. Nikoobakht, and M. A. El-Sayed, J. Phys. Chem. B 104, 6152 (2000).

30 E. Lukianova-Hleb, L. J. E. Anderson, S. Lee, J. H. Hafner, and D. O. Lapotko, Phys. Chem. Chem. Phys. 12, 12237 (2010).

31 A. Plech, V. Kotaidis, S. Grésillon, C. Dahmen, and G. von Plessen, Phys. Rev. B 70, 195423 (2004).

32 E. Sassaroli, K. C. P. Li, and B. E. O'Neill, Phys. Med. Biol. 54, 5541 (2009).

33 E. Y. Hleb and D. O. Lapotko, Nanotechology 19, 355702 (2008).

34 O. Ekici, R. K. Harrison, N. J. Durr, D. S. Eversole, M. Lee, and A. Ben-Yakar, J. Phys. D: Appl. Phys. 41, 185501 (2008).

35 M. Hu, H. Petrova, and G. V. Hartland, Chem. Phys. Lett. 391, 220 (2004).

36 S. Merabia, P. Keblinski, L. Joly, L. J. Lewis, and J. L. Barrat, Phys. Rev. E 79, 021404 (2009).

37 S. Merabia, S. Shenogin, L. Joly, P. Keblinski, and J. L. Barrat, PNAS 106, 15113 (2009).

38 A. J. Schmidt, J. D. Alper, M. Chiesa, G. Chen, S. K. Das, and K. Hamad-Schifferli, J. Phys. Chem. C 112, 13320 (2008).

39 O. M. Wilson, X. Hu, D. G. Cahill, and P. V. Braun, Phys. Rev. B 66, 224301 (2002).

40 Z. Ge, D. G. Cahill, and P. V. Braun, J. Phys. Chem. B 108, 18870 (2010).

41 J. Alper and K. Hamad-Schifferli, Langmuir 26, 3786 (2010).

42 Handbook of Physics (Springer, 2000).

43 J. C. Butcher, Numerical methods for ordinary differential equations (John Wiley \& Sons, 2003).

${ }^{44}$ G. Baffou, R. Quidant, and C. Girard, Phys. Rev. B 82, 165424 (2010).

${ }^{45}$ P. K. Jain, K. S. Lee, I. H. El-Sayed, and M. A. El-Sayed, J. Phys. Chem. B 110, 7238 (2006), ISSN 1520-6106. 
46 C. F. Bohren and D. R. Huffman, Absorption and scattering of light by small particles (Wiley interscience, 1983).

47 P. Grua, J. P. Morreeuw, H. Bercegol, G. Jonusauskas, and F. Vallée, Phys. Rev. B 68, 035424 (2003).

${ }^{48}$ H. Inouye, K. Tanaka, I. Tanahashi, and K. Hirao, Phys. Rev. B 57, 11334 (1998).

49 A. Arbouet, C. Voisin, D. Christofilos, P. Langot, N. Del Fatti, F. Vallée, J. Lermé, G. Celep, E. Cottancin, M. Gaudry, M. Pellarin, M. Broyer, M. Maillard, M. P. Pileni, and M. Treguer, Phys. Rev. Lett. 90, 177401 (2003).

50 W. Huang, W. Qian, M. A. El-Sayed, Y. Ding, and Z. L. Wang, J. Phys. Chem. C 111, 10751 (2007).

51 J. H. Hodak, A. Henglein, and G. V. Hartland, J. Chem. Phys. 111, 8613 (1999).

52 S. Link, C. Burda, Z. L. Wang, and M. A. El-Sayed, J. Chem. Phys. 111, 1255 (1999).

${ }^{53}$ G. Baffou, R. Quidant, and C. Girard, Appl. Phys. Lett. 94, 153109 (2009).

\section{Appendix A: Numerical algorithm}

In this section, we explain and detail how the physical system was modeled using a finite difference method (FDM) and in particular what the RK4 algorithm consists in.

We shall specifically use in this section the equations and the formalism based on dimensionless space and time variables $(\rho$ and $\tau)$ and dimensionless constants and parameters $\left(\beta, \gamma\right.$ and $\left.\lambda_{\mathrm{K}}\right)$.

\section{Model assuming a uniform NP temperature}

We consider in this part a spherical NP endowed with a surface conductivity $g$ and characterized by a uniform inner temperature $T_{\mathrm{NP}}(\tau)$. This problem is described by the set of equations (37). Since the NP temperature is assumed to be uniform, only the surrounding medium has to be meshed (see Fig. 9). The spatio-temporal meshing of the system is such as:

$$
\begin{aligned}
\rho_{i} & \equiv 1+i \times \delta \rho & i \in[0, N] \\
\tau_{j} & \equiv j \times \delta \tau & j \in[0, M] \\
T_{i, j} & \equiv T\left(\rho_{i}, \tau_{j}\right) &
\end{aligned}
$$

To simplify the notations and the explanations, we consider here a regular spatio-temperal mesh. However it would be wise to use for instance a refined mesh close the NP interface and a rougher mesh further.

The boundary condition (37) at the NP interface reads:

$$
-\frac{T_{2, j}-T_{1, j}}{\delta \rho}=\frac{T_{0, j}-T_{1, j}}{\lambda_{\mathrm{K}}}
$$

The temperature drop $\Delta T$ occurs between the coordinates $\rho_{0}$ and $\rho_{1}$. The temperature gradient on the NP surface is calculated between the coordinates $\rho_{1}$ and $\rho_{2}$ (see Fig. 9).

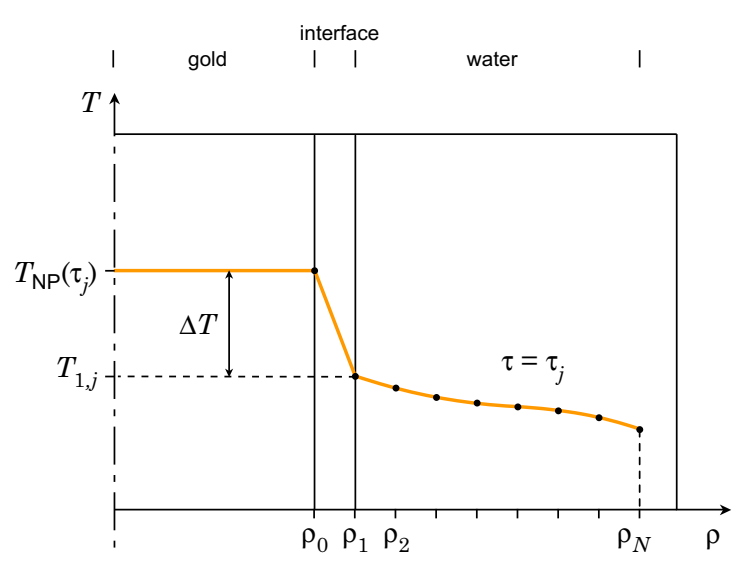

FIG. 9. Schematic of the temperature profile at time $\tau_{j}$ around a spherical gold nanoparticle as modeled in the FDMRK4 algorithm. In this first model, the NP temperature $T_{\mathrm{NP}}\left(\tau_{j}\right)$ is assumed to be uniform and a temperature discontinuity $\Delta T$ occurs at the nanoparticle interface to take into account a finite interface conductivity.

The initial temperature profile is set to:

$$
\begin{aligned}
& T_{0,0}=T_{\mathrm{NP}}^{0} \\
& T_{1,0}=\frac{T_{\mathrm{NP}}^{0}}{1+\delta \rho / \lambda_{\mathrm{K}}} \\
& T_{i, 0}=0 \quad \text { for } i>1
\end{aligned}
$$

This initial temperature profile corresponds to a zero temperature outside the NP and fulfills the boundary condition (A1).

The numerical algorithm consists in a spatial loop over the position $i$ inside a temporal loop over the time $j$. At each time $j+1$, here is the procedure.

First, the NP temperature $T_{0, j+1}$ is calculated from the second equation of system (37):

$$
T_{0, j+1}=T_{0, j}+3 \beta \delta \tau \frac{T_{2, j}-T_{1, j}}{\delta \rho} .
$$

Then, using Eq. (A1), one can compute the temperature on the NP surface $(i=1)$ :

$$
T_{1, j+1}=\frac{l_{\mathrm{K}} T_{2, j}+\delta \rho T_{0, j+1}}{\lambda_{\mathrm{K}}+\delta \rho} .
$$

Finally, the computation of the temperature profile in the surrounding medium $(i>2)$ is performed according to the RK4 procedure: ${ }^{43}$

$$
T_{i, j+1}=T_{i, j}+\frac{k_{1}+2 k_{2}+2 k_{3}+k_{4}}{6}
$$

where

$$
\begin{aligned}
& k_{1}=\delta t K\left(T_{i, j}\right) \\
& k_{2}=\delta t K\left(T_{i, j}+k_{1} / 2\right) \\
& k_{3}=\delta t K\left(T_{i, j}+k_{2} / 2\right) \\
& k_{4}=\delta t K\left(T_{i, j}+k_{3}\right)
\end{aligned}
$$

and

$$
K\left(T_{i, j}\right)=\frac{2}{\rho} \frac{T_{i+1, j}-T_{i, j}}{\delta \rho}+\frac{T_{i+1, j}-2 T_{i, j}+T_{i-1, j}}{\delta \rho^{2}} .
$$




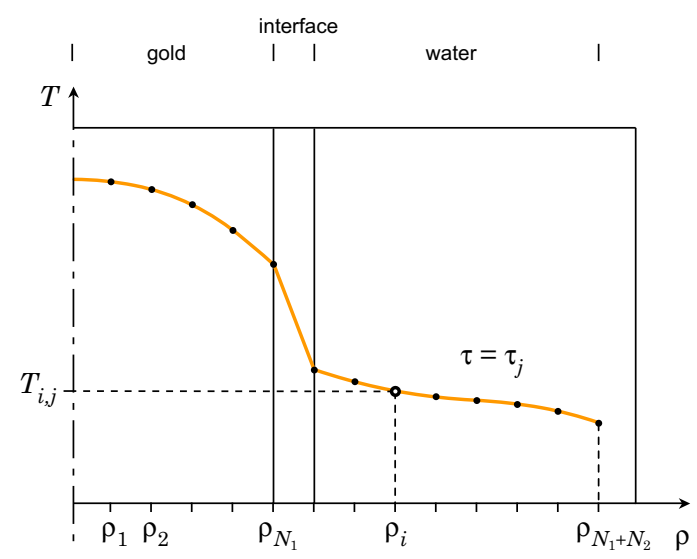

FIG. 10. Schematic of the temperature profile at time $\tau_{j}$ around a spherical gold nanoparticle as modeled in the FDMRK4 algorithm. In this second model, the temperature inside the NP is also meshed.

Note that the increments $\delta \rho$ and $\delta \tau$ cannot be chosen arbitrarily and independently. To ensure a proper convergence, $\delta \rho$ and $\delta \tau$ have to be chosen much smaller than unity and respecting as well the convergence criteria:

$$
\delta \rho^{2} / \delta \tau \gg 1 \text {. }
$$

This ensures that the right hand member of equation (A7) - the variation of the NP temperature - is small compared to the NP temperature $T_{i, j}$.

It is worth estimating the total energy of the system and its conservation at each time step to check the consistency of the calculations. The normalized energy at time $\tau_{j}$ expressed from equation (41) reads:

$$
\epsilon_{j}=\frac{T_{0, j}}{T_{0,0}}+3 \beta \delta \rho \sum_{i=1}^{N} \rho_{i}^{2} \frac{T_{i, j}}{T_{0,0}}
$$

and should remain close to unity at any step $j$ of the numerical procedure.

\section{Model including a non-uniform NP temperature}

We assume in this section that the inner temperature of the NP is not necessarily uniform. We shall also explain how both the heating and cooling of the NP can be investigated numerically.

In this context, the normalized coordinate reads now:

$$
\rho_{i} \equiv i \times \delta \rho \quad i \in\left[0, N_{1}+N_{2}-1\right]
$$

and in particular $\rho_{N_{1}}=N_{1} \times \delta \rho=1 . N_{1}$ is the number of mesh points in the NP and $N_{2}$ in the surrounding medium (see Fig. 10).

To simplify the notations and the explanations, we consider here a regular mesh. However it would make sense to use for instance a rough mesh inside the NP and a refined mesh in the surrounding medium, close to the NP interface.

The discretized boundary conditions of system (12) yields this time:

$\frac{T_{N_{1}+2, j}-T_{N_{1}+1, j}}{\delta \rho}=-\frac{T_{N_{1}, j}-T_{N_{1}+1, j}}{\lambda_{\mathrm{K}}}=\gamma \frac{T_{N_{1}, j}-T_{N_{1}-1, j}}{\delta \rho}$
At time $\tau_{0}$, the initial temperature profile is set to zero:

$$
\forall i, \quad T_{i, 0}=0 .
$$

At time $\tau_{j+1}$, the calculation of the temperature profile inside the NP $\left(i<N_{1}\right)$ is performed using the RK procedure (Eq. (A7)) and replacing the RK function $K\left(T_{i, j}\right)$ with

$$
K_{\mathrm{Au}}\left(T_{i, j}\right)=\beta \gamma K\left(T_{i, j}\right)+p_{0}\left(\tau_{j}\right) .
$$

This source term $p_{0}\left(\tau_{j}\right)$ has to be added when one wants to take into account a heat generation in the NP. For a pulsed illumination, it reads:

$$
p_{0}\left(\tau_{j}\right)=T_{\mathrm{NP}}^{0} \frac{\tau_{\mathrm{p}}}{\tau_{\mathrm{e}-\mathrm{ph}}} \exp \left(-\tau_{j} \tau_{\mathrm{p}} / \tau_{\mathrm{e}-\mathrm{ph}}\right) .
$$

And for a cw illumination:

$$
p_{0}=3 \beta T_{\mathrm{NP}}^{\mathrm{cW}} .
$$

The convergence criteria is now:

$$
\delta \rho^{2} / \delta \tau \gg \beta \gamma \approx 860 .
$$

This means that while investigating the dynamics of the NP temperature, the convergence criteria is more drastic. However, this is expected and not an issue since the associated time scale of the temperature diffusion inside the NP is much faster. Consequently, $\delta \tau$ can be chosen much smaller.

Then the temperatures at the nanoparticle interface $T_{N_{1}, j}$ and $T_{N_{1}+1, j}$ have to be calculated using the boundary conditions (A11). It yields:

$$
\begin{aligned}
T_{N_{1}, j+1} & =\frac{\gamma\left(\delta \rho+\lambda_{\mathrm{K}}\right) T_{N_{1}-1, j}+\delta \rho T_{N_{1}+2, j}}{\gamma \lambda_{\mathrm{K}}+\delta \rho+\gamma \delta \rho}, \\
T_{N_{1}+1, j+1} & =T_{N_{1}+2, j}+\gamma\left(T_{N_{1}-1, j}-T_{N_{1}, j+1}\right) .
\end{aligned}
$$

Finally the temperature profile within the surrounding medium $\left(i>N_{1}+1\right)$ is computed using the RK4 procedure (Eq. (A7)) and the regular RK function $K\left(T_{i, j}\right)$ (Eq. (A8)).

When considering an initial zero temperature profile, the new energy conservation laws (44) and (45) reads for $\mathrm{cw}$ illumination:

$$
3 \beta T_{\mathrm{NP}}^{\mathrm{wc}} j \delta \tau=\sum_{i=0}^{N_{1}} 3 \rho_{i}^{2} T_{i, j} \delta \rho+\sum_{i=N_{1}+1}^{N_{1}+N_{2}} 3 \beta \rho_{i}^{2} T_{i, j} \delta \rho
$$

And with pulsed illumination:

$$
\begin{aligned}
T_{\mathrm{NP}}^{0}\left[1-\exp \left(\frac{-\tau_{j} \tau_{\mathrm{d}}^{\mathrm{w}}}{\tau_{\mathrm{e}-\mathrm{ph}}}\right)\right] & =\sum_{i=0}^{N_{1}} 3 \rho_{i}^{2} T_{i, j} \delta \rho \\
& +\sum_{i=N_{1}+1}^{N_{1}+N_{2}} 3 \beta \rho_{i}^{2} T_{i, j} \delta \rho .
\end{aligned}
$$

